\title{
Bounding the integral of powered $i$-th mean curvatures
}

\author{
David Alonso-Gutiérrez, María A. Hernández Cifre and \\ Antonio R. Martínez Fernández
}

\begin{abstract}
We get estimates for the integrals of powered $i$-th mean curvatures, $1 \leq i \leq n-1$, of compact and convex hypersurfaces, in terms of the quermaßintegrals of the corresponding $C_{+}^{2}$ convex bodies. These bounds will be obtained as consequences of a most general result for functions defined on a general probability space. From this result, similar estimates for the integrals of any convex transformation of the elementary symmetric functions of the radii of curvature of $C_{+}^{2}$ convex bodies will be also proved, both, in terms of the quermaßintegrals, and of the roots of their Steiner polynomials. Finally, the radial function is considered, and estimates of the corresponding integrals are obtained in terms of the dual quermaßintegrals.
\end{abstract}

\section{Introduction and main results}

Let $\mathcal{K}_{0}^{n}$ be the set of all convex bodies, i.e., compact convex sets with non-empty interior, in the $n$-dimensional Euclidean space $\mathbb{R}^{n}$, containing the origin. Let $\langle\cdot, \cdot\rangle$ and $\|\cdot\|$ be the standard inner product and Euclidean norm in $\mathbb{R}^{n}$, respectively. The boundary of a set $M \subset \mathbb{R}^{n}$ is denoted by bd $M$ and its convex hull by conv $M$. We write $B_{2}^{n}$ to denote the Euclidean unit ball centered at the origin, $B_{2}^{n}(r)$ for the ball (centered at 0 ) with radius $r>0$, and $\mathbb{S}^{n-1}=\left\{x \in \mathbb{R}^{n}:\|x\|=1\right\}$ for the unit sphere in $\mathbb{R}^{n}$. Let $\mathrm{e}_{i}$ denote the $i$-th canonical unit vector.

A convex body $K \in \mathcal{K}_{0}^{n}$ is said to be of class $C^{2}$ if its boundary hypersurface bd $K$ is a regular submanifold of $\mathbb{R}^{n}$, in the sense of differential geometry, which is twice continuously differentiable. Moreover, we say that $K$ is of class $C_{+}^{2}$ if $K$ is of class $C^{2}$ and the Gauss map $\nu_{K}:$ bd $K \longrightarrow \mathbb{S}^{n-1}$, mapping a boundary point $x \in \mathrm{bd} K$ to the (unique) normal vector of $K$ at $x$, is a diffeomorphism.

Mathematics Subject Classification (2010): Primary 52A40, 05D40, 53C42; Secondary 52A22. Keywords: Convex hypersurfaces; $C_{+}^{2}$ convex bodies; mean curvatures; symmetric functions of radii of curvature; quermaßintegrals; inner and outer radii; roots of Steiner polynomials; radial function; dual quermaßintegrals. 
If $M$ is a (proper) Borel subset of $\mathbb{R}^{n}$, we will use the same notation $|\cdot|$ for its volume (i.e., its Lebesgue measure) and its surface area, writing $|M|$ and $|\mathrm{bd} M|$, respectively. Moreover, by $\mathcal{H}^{k}, 0 \leq k \leq n$, we denote the $k$-dimensional Hausdorff measure on $\mathbb{R}^{n}$, and thus, if $M$ is a subset of a $k$-plane or a $k$-dimensional sphere, then $\mathcal{H}^{k}(M)$ coincides, respectively, with the $k$-dimensional Lebesgue measure of $M$ in $\mathbb{R}^{k}$ or with the $k$-dimensional spherical Lebesgue measure in $\mathbb{S}^{k}$. Moreover, the outer radius and the inner radius of $K \in \mathcal{K}_{0}^{n}$ are defined as the quantities

$$
\begin{aligned}
\overline{\mathrm{R}}(K) & =\min \left\{R>0: K \subseteq R B_{2}^{n}\right\}=\max \{\|x\|: x \in \operatorname{bd} K\}, \\
\overline{\mathrm{r}}(K) & =\max \left\{r \geq 0: r B_{2}^{n} \subseteq K\right\}=\min \{\|x\|: x \in \operatorname{bd} K\} .
\end{aligned}
$$

Clearly, the value $\overline{\mathrm{R}}(K)-\overline{\mathrm{r}}(K)$ is not translation invariant, but since $K$ is compact, there exists a (unique) point $c_{K} \in K$ such that

$$
\overline{\mathrm{R}}\left(K-c_{K}\right)-\overline{\mathrm{r}}\left(K-c_{K}\right)=\min \{\overline{\mathrm{R}}(K-t)-\overline{\mathrm{r}}(K-t): t \in K\}
$$

(see [2]). We observe that $c_{K}$ is the so-called center of the minimal annulus, i.e., the uniquely determined annulus (closed set consisting of all points between two concentric balls) with minimal difference of radii containing bd $K$.

If $K$ is 0 -symmetric, i.e., if $K=-K$, then $\overline{\mathrm{R}}(K)$ and $\overline{\mathrm{r}}(K)$ coincide with the classical circumradius and inradius of $K$, namely,

$$
\begin{aligned}
\mathrm{R}(K) & =\min \left\{R: \exists x \in \mathbb{R}^{n} \text { with } K \subseteq x+R B_{2}^{n}\right\}, \\
\mathrm{r}(K) & =\max \left\{R: \exists x \in \mathbb{R}^{n} \text { with } x+R B_{2}^{n} \subseteq K\right\},
\end{aligned}
$$

respectively. In general, we say that a convex body $K$ is centered if $\mathrm{R}(K)=\overline{\mathrm{R}}(K)$ and $\mathrm{r}(K)=\overline{\mathrm{r}}(K)$.

Given a compact (oriented) hypersurface $M \subset \mathbb{R}^{n}$ with smooth boundary and mean curvature $H$ positive everywhere, Willmore's inequality says that

$$
\int_{M} H^{n-1} \mathrm{~d} \mathcal{H}^{n-1} \geq n\left|B_{2}^{n}\right|
$$

This was proved by Willmore [20] in the 3-dimensional case and by Chen in an arbitrary dimension, see $[4,5]$. Moreover, Ros [16] proved that

$$
\int_{M} \frac{1}{H} \mathrm{~d} \mathcal{H}^{n-1} \geq n|M|
$$

Besides the major importance that these results have by themselves, they are specially interesting because they imply isoperimetric inequalities (see e.g. [15]). Further information and related results on this topic can be found in, e.g., $[1,12]$ and the references given there. These inequalities have been also considered in a more general setting in, e.g., [14].

The proof of (1.1) uses as a key point the relation $H^{n-1} \geq \kappa$, where $\kappa$ denotes the Gauss-Kronecker curvature, being a trivial consequence of it in the particular case of a convex body $K \in \mathcal{K}_{0}^{n}$ of class $C_{+}^{2}$ (i.e., the hypersurface bd $K$ is convex). 
However, a natural problem would be to get improvements of (1.1) and (1.2) when we remain in the convex case. At this respect, in [8] the planar case is considered, and lower bounds for the integrals of powers of the curvature of a smooth bounded planar convex curve are shown.

One aim of the paper is to get estimates for the integrals of powered $i$-th mean curvatures $H_{i}, i=1, \ldots, n-1$, of compact and convex hypersurfaces, which include, in particular, the mean curvature $(i=1)$ and the Gauss-Kronecker curvature $(i=n-1)$. The bounds will be given in terms of the radii $\overline{\mathrm{r}}, \overline{\mathrm{R}}$ and the quermaßintegrals $\mathrm{W}_{j}$ of the corresponding convex bodies, which are special geometric measures associated to the set and including, in particular, the volume and the surface area (see Section 3 for the precise definition and properties).

Theorem 1.1. If $K \in \mathcal{K}_{0}^{n}$ is of class $C_{+}^{2}$ then, for any $\alpha \geq 0$ and all $0 \leq i \leq n-1$,

$$
\begin{gathered}
\int_{\mathrm{bd} K} H_{i}^{\alpha+1} \mathrm{~d} \mathcal{H}^{n-1} \geq \frac{n^{\alpha+1}}{2}\left[\frac{\mathrm{W}_{i+1}^{\alpha+1}}{\left(|\mathrm{bd} K|+\frac{\xi_{i}}{\mathrm{~W}_{i+1}}\right)^{\alpha}}+\frac{\mathrm{W}_{i+1}^{\alpha+1}}{\left(|\mathrm{bd} K|-\frac{\xi_{i}}{\mathrm{~W}_{i+1}}\right)^{\alpha}}\right], \\
\int_{\mathrm{bd} K} \frac{1}{H_{i}^{\alpha}} \mathrm{d} \mathcal{H}^{n-1} \geq \frac{1}{2 n^{\alpha}}\left[\frac{|\mathrm{bd} K|^{\alpha+1}}{\left(\mathrm{~W}_{i+1}+\frac{\xi_{i}}{|\mathrm{bd} K|}\right)^{\alpha}}+\frac{|\mathrm{bd} K|^{\alpha+1}}{\left(\mathrm{~W}_{i+1}-\frac{\xi_{i}}{|\mathrm{bd} K|}\right)^{\alpha}}\right]
\end{gathered}
$$

where

$$
\xi_{i}= \begin{cases}\frac{|\mathrm{bd} K| \mathrm{W}_{i}-n|K| \mathrm{W}_{i+1}}{\overline{\mathrm{R}}\left(K-c_{K}\right)-\overline{\mathrm{r}}\left(K-c_{K}\right)} & \text { if } K \neq B_{2}^{n}(r) \text { for any } r>0 \\ 0 & \text { if } K=B_{2}^{n}(r) \text { for some } r>0\end{cases}
$$

Equality holds in both inequalities if $K=B_{2}^{n}$ (up to dilations).

In particular, lower bounds for the integrals of the square/ $(n-1)$-power and the inverse of the classical mean and Gauss-Kronecker curvatures can be obtained:

Corollary 1.2. Let $K \in \mathcal{K}_{0}^{n}$ be of class $C_{+}^{2}$. Then,

$$
\begin{aligned}
& \int_{\mathrm{bd} K} H^{n-1} \mathrm{~d} \mathcal{H}^{n-1} \geq \frac{n^{n-1}}{2}\left[\frac{\mathrm{W}_{2}^{n-1}}{\left(|\mathrm{bd} K|+\frac{\xi_{1}}{\mathrm{~W}_{2}}\right)^{n-2}}+\frac{\mathrm{W}_{2}^{n-1}}{\left(|\mathrm{bd} K|-\frac{\xi_{1}}{\mathrm{~W}_{2}}\right)^{n-2}}\right] \\
& \int_{\mathrm{bd} K} \frac{1}{H} \mathrm{~d} \mathcal{H}^{n-1} \geq \frac{1}{n} \frac{|\mathrm{bd} K|^{2} \mathrm{~W}_{2}}{\mathrm{~W}_{2}^{2}-\frac{\xi_{1}^{2}}{|\mathrm{bd} K|^{2}}} \text { and } \\
& \int_{\mathrm{bd} K} H^{2} \mathrm{~d} \mathcal{H}^{n-1} \geq n^{2} \frac{|\mathrm{bd} K| \mathrm{W}_{2}^{2}}{|\mathrm{bd} K|^{2}-\frac{\xi_{1}^{2}}{\mathrm{~W}_{2}^{2}}} .
\end{aligned}
$$

Equality holds in all inequalities if $K=B_{2}^{n}$ (up to dilations). 
The first and second bounds, as well as the ones obtained with the tighter inequalities given in Theorems 3.3 and 3.4, improve the known ones for convex hypersurfaces given by (1.1) and (1.2) (see Remark 3.5).

Corollary 1.3. Let $K \in \mathcal{K}_{0}^{n}$ be of class $C_{+}^{2}$. Then,

$$
\begin{gathered}
\int_{\mathrm{bd} K} \kappa^{2} \mathrm{~d} \mathcal{H}^{n-1} \geq n^{2} \frac{\left|B_{2}^{n}\right|^{2}|\mathrm{bd} K|}{|\mathrm{bd} K|^{2}-\frac{\xi_{n-1}^{2}}{\left|B_{2}^{n}\right|^{2}}}, \\
\int_{\mathrm{bd} K} \frac{1}{\kappa} \mathrm{d} \mathcal{H}^{n-1} \geq \frac{1}{n} \frac{\left|B_{2}^{n}\right||\mathrm{bd} K|^{2}}{\left|B_{2}^{n}\right|^{2}-\frac{\xi_{n-1}^{2}}{|\mathrm{bd} K|^{2}}} .
\end{gathered}
$$

Equality holds in both inequalities if $K=B_{2}^{n}$ (up to dilations).

These higher dimensional extensions will be consequences of a very general result for functions defined on a general probability space, which will be obtained following the ideas in [8]. More precisely, we will prove the following proposition.

Proposition 1.4. Let $(\Omega, \mathbb{P})$ be a probability space such that, for any $A \subseteq \Omega$ and any $0 \leq p \leq \mathbb{P}(A)$, there exists $B \subseteq A$ with $\mathbb{P}(B)=p$. Let $\rho, h: \Omega \longrightarrow \mathbb{R}$, with $\rho \in L^{1}(\Omega)$ and $h \in L^{\infty}(\Omega)$. Then, for any convex function $F: I \longrightarrow \mathbb{R}, I \subseteq \mathbb{R}$ where all the expressions below are defined, we have

$$
\mathbb{E}(F \circ \rho) \geq \frac{F\left(\mathbb{E} \rho+\frac{\operatorname{Cov}(\rho, h)}{\|h-\mathbb{E} h\|_{\infty}}\right)+F\left(\mathbb{E} \rho-\frac{\operatorname{Cov}(\rho, h)}{\|h-\mathbb{E} h\|_{\infty}}\right)}{2} .
$$

Here, as usual in the literature,

$$
\mathbb{E} \rho=\int_{\Omega} \rho(\omega) \mathrm{d} \mathbb{P}(\omega)
$$

will denote the expectation of $\rho, \operatorname{Cov}(\rho, h)=\mathbb{E} h \rho-\mathbb{E} h \mathbb{E} \rho$ the covariance of $\rho$ and $h$, and $\|\cdot\|_{\infty}$ the sup-norm, i.e., $\|f\|_{\infty}=\sup \{|f(\omega)|: \omega \in \Omega\}$.

The paper is organized as follows. In Section 2 we study the probabilistic type results that will be the key to the later development; in particular, we prove Proposition 1.4. Then Section 3 is devoted to provide tight estimates for the integral of any convex function of the $i$-th mean curvatures $H_{i}$ (Theorem 3.2), which will allow to get, as particular cases, lower bounds for the integral of any power of $H_{i}$ and $1 / H_{i}$ (Theorems 3.3 and 3.4). Other consequences, such as an estimate for the entropy of $H_{i}$, are also obtained.

Analogous results to the ones of the $H_{i}$ 's, but for the elementary symmetric functions of the radii of curvature of a $C_{+}^{2}$ convex body, are proved in Section 4 (Theorem 4.1); additional considerations regarding the possibility of removing the inner and outer radii from the bounds are also made. Then, Section 5 is devoted to relate the integral of the symmetric functions of the radii of curvature, to the roots of the Steiner polynomial of the convex body, as well as to consider particular families of bodies like constant width sets. Finally, in Section 6, we provide tight estimates for the integral of any convex function of (powers of) the radial function of a convex body. In this case, the bounds will be given in terms of the so-called dual quermaßintegrals. 


\section{Some probabilistic type results}

In this section we show the probabilistic results (Proposition 1.4 and, as a consequence, Proposition 2.2) from which all theorems will be obtained.

We start with the proof of Proposition 1.4.

Proof of Proposition 1.4. Without loss of generality we assume that $\operatorname{Cov}(\rho, h) \leq 0$; otherwise we just change $h$ by $-h$.

Let $m$ be a median of $\rho$, i.e., a value for which both $\mathbb{P}(\{\omega \in \Omega: \rho(\omega) \geq m\}) \geq$ $1 / 2$ and $\mathbb{P}(\{\omega \in \Omega: \rho(\omega) \leq m\}) \geq 1 / 2$, and let $\Omega_{1} \subset \Omega$ and $\Omega_{2}=\Omega \backslash \Omega_{1}$ be such that $\mathbb{P}\left(\Omega_{1}\right)=\mathbb{P}\left(\Omega_{2}\right)=1 / 2$ and

$$
\begin{aligned}
& \{\omega \in \Omega: \rho(\omega)>m\} \subseteq \Omega_{1} \subseteq\{\omega \in \Omega: \rho(\omega) \geq m\} \\
& \{\omega \in \Omega: \rho(\omega)<m\} \subseteq \Omega_{2} \subseteq\{\omega \in \Omega: \rho(\omega) \leq m\}
\end{aligned}
$$

We notice that such $\Omega_{1}$ always exists. Indeed, by the definition of median,

$$
\mathbb{P}(\{\omega \in \Omega: \rho(\omega) \leq m\}) \geq \frac{1}{2} \quad \text { and so } \quad \mathbb{P}(\{\omega \in \Omega: \rho(\omega)>m\}) \leq \frac{1}{2}
$$

Consequently, since

$\mathbb{P}(\{\omega \in \Omega: \rho(\omega) \geq m\})=\mathbb{P}(\{\omega \in \Omega: \rho(\omega)>m\})+\mathbb{P}(\{\omega \in \Omega: \rho(\omega)=m\}) \geq \frac{1}{2}$,

we have that

$$
\mathbb{P}(\{\omega \in \Omega: \rho(\omega)=m\}) \geq \frac{1}{2}-\mathbb{P}(\{\omega \in \Omega: \rho(\omega)>m\}) \geq 0 .
$$

Then, by our assumptions on $(\Omega, \mathbb{P})$, there exists a subset $B \subseteq\{\omega \in \Omega: \rho(\omega)=m\}$ with $\mathbb{P}(B)=1 / 2-\mathbb{P}\{\omega \in \Omega: \rho(\omega)>m\}$ and then we can take

$$
\Omega_{1}=\{\omega \in \Omega: \rho(\omega)>m\} \cup B .
$$

Now, let

$$
\rho_{1}=2 \int_{\Omega_{1}} \rho(\omega) \mathrm{d} \mathbb{P}(\omega) \quad \text { and } \quad \rho_{2}=2 \int_{\Omega_{2}} \rho(\omega) \mathrm{d} \mathbb{P}(\omega) .
$$

Since $\rho_{1}+\rho_{2}=2 \mathbb{E} \rho$, we can write

$$
\rho_{1}=\mathbb{E} \rho+b \quad \text { and } \quad \rho_{2}=\mathbb{E} \rho-b
$$

for some $b \geq 0$. First, we are going to prove that

$$
\frac{|\operatorname{Cov}(\rho, h)|}{\|h-\mathbb{E} h\|_{\infty}} \leq b
$$

Indeed, since

$$
-\|h-\mathbb{E} h\|_{\infty} \leq \mathbb{E} h-h(\omega) \leq\|h-\mathbb{E} h\|_{\infty}
$$


for every $\omega \in \Omega$ and since $\rho(\omega) \geq m$ if $\omega \in \Omega_{1}$ and $\rho(\omega) \leq m$ if $\omega \in \Omega_{2}$, then we have that

$$
\int_{\Omega_{1}}(\mathbb{E} h-h(\omega))(\rho(\omega)-m) \mathrm{d} \mathbb{P}(\omega) \leq \frac{1}{2}\|h-\mathbb{E} h\|_{\infty}\left(\rho_{1}-m\right)
$$

and

$$
\int_{\Omega_{2}}(\mathbb{E} h-h(\omega))(\rho(\omega)-m) \mathrm{d} \mathbb{P}(\omega) \leq-\frac{1}{2}\|h-\mathbb{E} h\|_{\infty}\left(\rho_{2}-m\right) .
$$

Adding both integrals and using (2.1) we get

$$
\begin{aligned}
\mathbb{E}((\mathbb{E} h-h)(\rho-m)) & =\int_{\Omega}(\mathbb{E} h-h(\omega))(\rho(\omega)-m) \mathrm{d} \mathbb{P}(\omega) \\
& \leq \frac{1}{2}\|h-\mathbb{E} h\|_{\infty}\left(\rho_{1}-\rho_{2}\right)=\|h-\mathbb{E} h\|_{\infty} b,
\end{aligned}
$$

and since

$$
\mathbb{E}((\mathbb{E} h-h)(\rho-m))=\mathbb{E} h \mathbb{E} \rho-\mathbb{E} h \rho=-\operatorname{Cov}(\rho, h),
$$

we obtain the required bound (2.2).

Now, since $F$ is convex, Jensen's inequality (see e.g. p. 20 in [17]) yields

$$
F\left(\rho_{1}\right) \leq 2 \int_{\Omega_{1}}(F \circ \rho)(\omega) \mathrm{d} \mathbb{P}(\omega) \quad \text { and } \quad F\left(\rho_{2}\right) \leq 2 \int_{\Omega_{2}}(F \circ \rho)(\omega) \mathrm{d} \mathbb{P}(\omega),
$$

which, together with (2.1) implies that

$$
\begin{aligned}
\mathbb{E}(F \circ \rho) & =\int_{\Omega_{1}}(F \circ \rho)(\omega) \mathrm{d} \mathbb{P}(\omega)+\int_{\Omega_{2}}(F \circ \rho)(\omega) \mathrm{d} \mathbb{P}(\omega) \\
& \geq \frac{F\left(\rho_{1}\right)+F\left(\rho_{2}\right)}{2}=\frac{F(\mathbb{E} \rho+b)+F(\mathbb{E} \rho-b)}{2} .
\end{aligned}
$$

Finally, since a convex function $F$ satisfies that for any $x \in \mathbb{R}$ and $0 \leq a \leq b$ the average of $\{F(x+a), F(x-a)\}$ is not bigger than the average of $\{F(x+b), F(x-b)\}$, taking into account $(2.2)$ we can conclude that

$$
\mathbb{E}(F \circ \rho) \geq \frac{F(\mathbb{E} \rho+b)+F(\mathbb{E} \rho-b)}{2} \geq \frac{F\left(\mathbb{E} \rho+\frac{\operatorname{Cov}(\rho, h)}{\|h-\mathbb{E} h\|_{\infty}}\right)+F\left(\mathbb{E} \rho-\frac{\operatorname{Cov}(\rho, h)}{\|h-\mathbb{E} h\|_{\infty}}\right)}{2} .
$$

An interesting particular case is obtained when $F(x)=1 / x$ :

Corollary 2.1. Let $(\Omega, \mathbb{P})$ be a probability space such that, for any $A \subseteq \Omega$ with positive probability and any $0 \leq p \leq \mathbb{P}(A)$, there exists $B \subseteq A$ with $\mathbb{P}(B)=p$. Let $\rho, h: \Omega \longrightarrow \mathbb{R}$, with $\rho \in L^{1}(\Omega)$ and $h \in L^{\infty}(\Omega)$. Then,

$$
\mathbb{E} \frac{1}{\rho} \geq \frac{\mathbb{E} \rho}{(\mathbb{E} \rho)^{2}-\frac{\operatorname{Cov}(\rho, h)^{2}}{\|h-\mathbb{E} h\|_{\infty}^{2}}}
$$


If we express the probability measure by means of a density with respect to another (not necessarily a probability) measure $\mu$, we immediately obtain the following result.

Proposition 2.2. Let $(\Omega, \mu)$ be a measure space and let $g: \Omega \longrightarrow \mathbb{R}$ be a positive integrable function such that $\int_{\Omega} g \mathrm{~d} \mu=1$, and for any $A \subseteq \Omega$ with $\int_{A} g \mathrm{~d} \mu>0$ and any $0 \leq p \leq \int_{A} g \mathrm{~d} \mu$, there exists $B \subseteq A$ with $\int_{B} g \mathrm{~d} \mu=p$. Let $\rho, h: \Omega \longrightarrow \mathbb{R}$ be integrable functions with $h \in L^{\infty}(\Omega)$. Then, for any convex function $F: I \longrightarrow \mathbb{R}$, $I \subseteq \mathbb{R}$ where all the expressions below are defined, we have

$$
\int_{\Omega}(F \circ \rho) g \mathrm{~d} \mu \geq \frac{F\left(\int_{\Omega} \rho g \mathrm{~d} \mu+\eta(\rho, h, g)\right)+F\left(\int_{\Omega} \rho g \mathrm{~d} \mu-\eta(\rho, h, g)\right)}{2},
$$

where

$$
\eta(\rho, h, g)=\frac{\int_{\Omega} \rho h g \mathrm{~d} \mu-\left(\int_{\Omega} \rho g \mathrm{~d} \mu\right)\left(\int_{\Omega} h g \mathrm{~d} \mu\right)}{\left\|h-\int_{\Omega} h g \mathrm{~d} \mu\right\|_{\infty}} .
$$

Rewriting the above proposition for some particular functions, special inequalities can be obtained. For instance, we can take $\rho=1 / g$ and, either $F(x)=1 / x^{\alpha}$ or $F(x)=x^{\alpha+1}$ with $\alpha \geq 0$ :

Corollary 2.3. Let $(\Omega, \mu)$ be a measure space and let $g: \Omega \rightarrow \mathbb{R}$ be a positive integrable function with $\int_{\Omega} g \mathrm{~d} \mu=1,1 / g$ integrable and such that, for any $A \subseteq \Omega$ with $\int_{A} g \mathrm{~d} \mu>0$ and any $0 \leq p \leq \int_{A} g \mathrm{~d} \mu$, there exists $B \subseteq A$ with $\int_{B} g \mathrm{~d} \mu=p$. Let $h: \Omega \longrightarrow \mathbb{R}$ be an integrable function, $h \in L^{\infty}(\Omega)$, and let

$$
\eta(h, g)=\frac{\int_{\Omega} h \mathrm{~d} \mu-\mu(\Omega) \int_{\Omega} h g \mathrm{~d} \mu}{\left\|h-\int_{\Omega} h g \mathrm{~d} \mu\right\|_{\infty}} .
$$

Then, for any $\alpha \geq 0$,

$$
\int_{\Omega} g^{\alpha+1} \mathrm{~d} \mu \geq \frac{1}{2}\left(\frac{1}{(\mu(\Omega)+\eta(h, g))^{\alpha}}+\frac{1}{(\mu(\Omega)-\eta(h, g))^{\alpha}}\right)
$$

and

$$
\int_{\Omega} \frac{1}{g^{\alpha}} \mathrm{d} \mu \geq \frac{(\mu(\Omega)+\eta(h, g))^{\alpha+1}+(\mu(\Omega)-\eta(h, g))^{\alpha+1}}{2}
$$

In particular, taking $\alpha=1$ in (2.4) and in (2.5), we get

$$
\int_{\Omega} g^{2} \mathrm{~d} \mu \geq \frac{\mu(\Omega)}{\mu(\Omega)^{2}-\eta(h, g)^{2}} \quad \text { and } \quad \int_{\Omega} \frac{1}{g} \mathrm{~d} \mu \geq \mu(\Omega)^{2}+\eta(h, g)^{2},
$$

respectively.

It is also of particular interest to obtain an estimate for the entropy of $g$, i.e., to consider $F(x)=-\log x$ and again $\rho=1 / g$. 
Corollary 2.4. Let $(\Omega, \mu)$ be a measure space and let $g: \Omega \longrightarrow \mathbb{R}$ be an integrable function with $\int_{\Omega} g \mathrm{~d} \mu=1,1 / g$ integrable and such that, for any $A \subseteq \Omega$ with $\int_{A} g \mathrm{~d} \mu>0$ and any $0 \leq p \leq \int_{A} g \mathrm{~d} \mu$, there exists $B \subseteq A$ with $\int_{B} g \mathrm{~d} \mu=p$. Let $h: \Omega \longrightarrow \mathbb{R}$ be an integrable function, $h \in L^{\infty}(\Omega)$. Then

$$
\int_{\Omega} g \log g \mathrm{~d} \mu \geq-\frac{1}{2} \log \left(\mu(\Omega)^{2}-\eta(h, g)^{2}\right),
$$

where $\eta(h, g)$ is defined as in (2.3).

\section{Mean curvatures of hypersurfaces}

For $K \in \mathcal{K}_{0}^{n}$ and a non-negative real number $\lambda$, the volume of the Minkowski sum (vectorial addition) $K+\lambda B_{2}^{n}$ is expressed as a polynomial of degree $n$ in $\lambda$, namely,

$$
\left|K+\lambda B_{2}^{n}\right|=\sum_{i=0}^{n}\left(\begin{array}{c}
n \\
i
\end{array}\right) \mathrm{W}_{i}(K) \lambda^{i},
$$

which is called the (classical) Steiner formula of $K$ (see [18]). The coefficients $\mathrm{W}_{i}(K)$ are the quermaßintegrals of $K$, and they are a special case of the more general defined mixed volumes for which we refer to Section 5.1 in [17]. In particular, $\mathrm{W}_{0}(K)=|K|$ (the area $\mathrm{A}(K)$ in the planar case), $n \mathrm{~W}_{1}(K)=\mid$ bd $K \mid$ (the perimeter $\mathrm{p}(K)$ in the plane) and $2 \mathrm{~W}_{n-1} /\left|B_{2}^{n}\right|=\mathrm{b}(K)$ is the mean width of $K$. Moreover, $\mathrm{W}_{n}(K)=\left|B_{2}^{n}\right|$, which takes the value

$$
\left|B_{2}^{n}\right|=\frac{\pi^{n / 2}}{\Gamma\left(\frac{n}{2}+1\right)},
$$

where $\Gamma$ is the gamma function. Then, denoting by B the beta function, it is easy to check the one-dimensional recursion formula (see e.g. Section 5.3 in[19])

$$
\frac{\left|B_{2}^{n}\right|}{\left|B_{2}^{n-1}\right|}=\frac{\pi^{1 / 2} \Gamma\left(\frac{n+1}{2}\right)}{\Gamma\left(\frac{n}{2}+1\right)}=\mathrm{B}\left(\frac{1}{2}, \frac{n+1}{2}\right)=2 \int_{0}^{1}\left(1-x^{2}\right)^{(n-1) / 2} \mathrm{~d} x .
$$

From now on, for the sake of brevity, we will write $\mathrm{W}_{i}=\mathrm{W}_{i}(K), i=0, \ldots, n$, and analogously for all radii, if the distinction of the body is not needed.

Further, for $1 \leq j \leq k \leq n-1,0 \leq l \leq n-1$, let

$$
\begin{aligned}
\eta_{j, k, l}= & \frac{\mathrm{W}_{j} \mathrm{~W}_{k}-\mathrm{W}_{j-1} \mathrm{~W}_{k+1}}{\mathrm{~W}_{l+1}^{2} \min _{x \in K} \max \left\{\overline{\mathrm{R}}(K-x)-\frac{\mathrm{W}_{l}}{\mathrm{~W}_{l+1}}, \frac{\mathrm{W}_{l}}{\mathrm{~W}_{l+1}}-\overline{\mathrm{r}}(K-x)\right\}} \\
& \text { if } K \neq B_{2}^{n}(r) \text { for any } r>0, \\
\eta_{j, k, l}= & 0 \quad \text { if } K=B_{2}^{n}(r) \text { for some } r>0 .
\end{aligned}
$$

We observe that since $\overline{\mathrm{r}} B_{2}^{n} \subseteq K$ and $K \subseteq \overline{\mathrm{R}} B_{2}^{n}$, the inequalities

$$
\overline{\mathrm{r}} \mathrm{W}_{i+1} \leq \mathrm{W}_{i} \leq \overline{\mathrm{R}} \mathrm{W}_{i+1},
$$


$i=0, \ldots, n-1$, are a direct consequence of the monotonicity of the mixed volumes (cf. e.g. p. 282 in [17]); hence the maximum in (3.3) is always positive (quermaßintegrals are translation invariant functionals). This, together with the inequalities

$$
\mathrm{W}_{j} \mathrm{~W}_{k} \geq \mathrm{W}_{j-1} \mathrm{~W}_{k+1}, \quad 1 \leq j \leq k \leq n-1
$$

which are particular cases of the Aleksandrov-Fenchel inequality (see e.g. Section 7.3 in [17]), ensures that $\eta_{j, k, l} \geq 0$.

Remark 3.1. At this point we would like to observe that the point $x \in K$ for which the minimum in (3.3) is attained and $c_{K}$ do not necessarily coincide, and thus, we cannot just assume that $c_{K}$ is the origin in all the results. Indeed, taking the triangle

$$
T=\operatorname{conv}\left\{(0,0)^{\top},(1,0)^{\top},\left(\frac{\sqrt{3}}{2}, \frac{1}{2}\right)^{\top}\right\},
$$

it is easy to see that $c_{T}=(1 / 2,(2-\sqrt{3}) / 2)^{\top}, \overline{\mathrm{R}}\left(T-c_{T}\right)=(\sqrt{6}-\sqrt{2}) / 2$ and $\overline{\mathrm{r}}\left(T-c_{T}\right)=(2-\sqrt{3}) / 2$, which yields

$$
\max \left\{\overline{\mathrm{R}}\left(T-c_{T}\right)-\frac{\mathrm{p}}{2 \pi}, \frac{\mathrm{p}}{2 \pi}-\overline{\mathrm{r}}\left(T-c_{T}\right)\right\}=\frac{\mathrm{p}}{2 \pi}-\overline{\mathrm{r}}\left(T-c_{T}\right)=0.2667 \ldots
$$

However, there are translation points $x \in T$ for which the corresponding maximum is smaller: for instance, if we take $x=(3 / 5,3(2-\sqrt{3}) / 5)^{\top}$, it can be checked that

$$
\max \left\{\overline{\mathrm{R}}(T-x)-\frac{\mathrm{p}}{2 \pi}, \frac{\mathrm{p}}{2 \pi}-\overline{\mathrm{r}}(T-x)\right\}=\frac{\mathrm{p}}{2 \pi}-\overline{\mathrm{r}}(T-x)=0.2399 \ldots
$$

The $C_{+}^{2}$ case follows by approximation.

Throughout the paper we will denote by

$$
\sigma_{i}\left(x_{1}, \ldots, x_{m}\right)=\sum_{1 \leq j_{1}<\cdots<j_{i} \leq m} x_{j_{1}} \cdot \ldots \cdot x_{j_{i}}
$$

the $i$-th elementary symmetric function of $x_{1}, \ldots, x_{m} \in \mathbb{R}, 1 \leq i \leq m$, setting $\sigma_{0}\left(x_{1}, \ldots, x_{m}\right)=1$. Furthermore, we will use $\mathrm{s}_{i}\left(x_{1}, \ldots, x_{m}\right)$ for the normalized $i$-th elementary symmetric function, i.e.,

$$
\mathrm{s}_{i}\left(x_{1}, \ldots, x_{m}\right)=\left(\begin{array}{c}
m \\
i
\end{array}\right)^{-1} \sum_{1 \leq j_{1}<\cdots<j_{i} \leq m} x_{j_{1}} \cdot \ldots \cdot x_{j_{i}},
$$

with $\mathrm{s}_{0}\left(x_{1}, \ldots, x_{m}\right)=1$.

If $K \in \mathcal{K}_{0}^{n}$ is of class $C_{+}^{2}$, then its boundary bd $K$ is a (compact) hypersurface of $\mathbb{R}^{n}$, and we can consider the $n-1$ principal curvatures $k_{1}, \ldots, k_{n-1}$ of bd $K$. As usual in the literature, by

$$
H_{i}=\mathrm{s}_{i}\left(k_{1}, \ldots, k_{n-1}\right), \quad i=1, \ldots, n-1,
$$


we denote the $i$-th mean curvature, setting $H_{0}=1$, and we refer to $H=H_{1}$ as the classical mean curvature and to $\kappa=H_{n-1}$ as the Gauss-Kronecker curvature. Then (see e.g. pp. 296-297 in [17]) it is known that, for $i=1, \ldots, n$,

$$
\mathrm{W}_{i}=\frac{1}{n} \int_{\mathrm{bd} K} H_{i-1} \mathrm{~d} \mathcal{H}^{n-1}=\frac{1}{n} \int_{\mathrm{bd} K} q_{K} H_{i} \mathrm{~d} \mathcal{H}^{n-1},
$$

where $q_{K}(x)=h_{K}\left(\nu_{K}(x)\right)$ for $x \in \operatorname{bd} K$, and $h_{K}(u)=\sup _{x \in K}\langle x, u\rangle, u \in \mathbb{R}^{n}$, is the support function of $K$ (see e.g. Section 1.7 in [17]). We observe that the volume

$$
|K|=\mathrm{W}_{0}=\frac{1}{n} \int_{\mathrm{bd} K} q_{K} H_{0} \mathrm{~d} \mathcal{H}^{n-1}=\frac{1}{n} \int_{\mathrm{bd} K} q_{K} \mathrm{~d} \mathcal{H}^{n-1} .
$$

First we show the following general result for an arbitrary convex function.

Theorem 3.2. Let $K \in \mathcal{K}_{0}^{n}$ be of class $C_{+}^{2}$. For any convex function $F: I \longrightarrow \mathbb{R}$, $I \subseteq \mathbb{R}$ where all the quantities are defined, and all $0 \leq i \leq n-1$,

$$
\int_{\mathrm{bd} K}\left(F \circ H_{i}\right) \mathrm{d} \mathcal{H}^{n-1} \geq n \mathrm{~W}_{1} \frac{F\left(\frac{\mathrm{W}_{i+1}}{\mathrm{~W}_{1}}+\eta_{1, i, 0}\right)+F\left(\frac{\mathrm{W}_{i+1}}{\mathrm{~W}_{1}}-\eta_{1, i, 0}\right)}{2},
$$

$$
\int_{\mathrm{bd} K}\left(F \circ \frac{1}{H_{i}}\right) H_{i} \mathrm{~d} \mathcal{H}^{n-1} \geq n \mathrm{~W}_{i+1} \frac{F\left(\frac{\mathrm{W}_{1}}{\mathrm{~W}_{i+1}}+\eta_{1, i, i}\right)+F\left(\frac{\mathrm{W}_{1}}{\mathrm{~W}_{i+1}}-\eta_{1, i, i}\right)}{2}
$$

Equality holds in both inequalities if $K=B_{2}^{n}$ (up to dilations).

Proof. In order to get (3.7), we consider the probability space (bd $K, \mathcal{H}^{n-1} /\left(n \mathrm{~W}_{1}\right)$ ) and apply Proposition 1.4 to the functions $\rho=H_{i}$ and $h=q_{K}$. Then, using the identities in (3.6), we get $\mathbb{E} \rho=\mathrm{W}_{i+1} / \mathrm{W}_{1}, \mathbb{E} h=\mathrm{W}_{0} / \mathrm{W}_{1}$ and

$$
\operatorname{Cov}(\rho, h)=\mathbb{E} h \rho-\mathbb{E} h \mathbb{E} \rho=\frac{\mathrm{W}_{i} \mathrm{~W}_{1}-\mathrm{W}_{0} \mathrm{~W}_{i+1}}{\mathrm{~W}_{1}^{2}} .
$$

Moreover,

$$
\|h-\mathbb{E} h\|_{\infty}=\sup \left\{\left|q_{K}(x)-\frac{\mathrm{W}_{0}}{\mathrm{~W}_{1}}\right|: x \in \operatorname{bd} K\right\}=\max \left\{\overline{\mathrm{R}}-\frac{\mathrm{W}_{0}}{\mathrm{~W}_{1}}, \frac{\mathrm{W}_{0}}{\mathrm{~W}_{1}}-\overline{\mathrm{r}}\right\},
$$

and since the functionals $H_{j}, \mathrm{~W}_{j}$ are translation invariant, the greatest possible lower bound for $\int_{\mathrm{bd} K}\left(F \circ H_{i}\right) \mathrm{d} \mathcal{H}^{n-1}$ will be obtained for the translation of $K$ such that the above maximum is minimal. Altogether gives (3.7).

Inequality (3.8) is obtained analogously, but now as a consequence of Proposition 2.2 for $\rho=1 / H_{i}, h=q_{K}$ and $g=H_{i} /\left(n \mathrm{~W}_{i+1}\right)$; we notice that, by (3.6), $\int_{\mathrm{bd} K} g \mathrm{~d} \mathcal{H}^{n-1}=1$.

Finally, equality trivially holds for $K=B_{2}^{n}$ (up to dilations) just noticing that $\mathrm{W}_{i}\left(B_{2}^{n}\right)=\left|B_{2}^{n}\right|$ for all $i=0, \ldots, n$ and $\left|\mathbb{S}^{n-1}\right|=n\left|B_{2}^{n}\right|$. 
As our main aim is to get bounds for the integral of powered mean curvatures, this may be achieved applying Theorem 3.2 to the functions $F(x)=x^{\alpha+1}$ or $F(x)=1 / x^{\alpha}, \alpha \geq 0$. Thus, two different results can be obtained, providing different bounds for the same integrals:

Theorem 3.3. Let $K \in \mathcal{K}_{0}^{n}$ be of class $C_{+}^{2}$. For all $0 \leq i \leq n-1$ and any $\alpha \geq 0$,

$$
\begin{aligned}
& \int_{\mathrm{bd} K} H_{i}^{\alpha+1} \mathrm{~d} \mathcal{H}^{n-1} \geq \frac{n}{2}\left[\frac{\left(\mathrm{W}_{i+1}+\mathrm{W}_{1} \eta_{1, i, 0}\right)^{\alpha+1}}{\mathrm{~W}_{1}^{\alpha}}+\frac{\left(\mathrm{W}_{i+1}-\mathrm{W}_{1} \eta_{1, i, 0}\right)^{\alpha+1}}{\mathrm{~W}_{1}^{\alpha}}\right], \\
& \int_{\mathrm{bd} K} \frac{1}{H_{i}^{\alpha}} \mathrm{d} \mathcal{H}^{n-1} \geq \frac{n}{2}\left[\frac{\left(\mathrm{W}_{1}+\mathrm{W}_{i+1} \eta_{1, i, i}\right)^{\alpha+1}}{\mathrm{~W}_{i+1}^{\alpha}}+\frac{\left(\mathrm{W}_{1}-\mathrm{W}_{i+1} \eta_{1, i, i}\right)^{\alpha+1}}{\mathrm{~W}_{i+1}^{\alpha}}\right] .
\end{aligned}
$$

Equality holds in both inequalities if $K=B_{2}^{n}$ (up to dilations).

Theorem 3.4. Let $K \in \mathcal{K}_{0}^{n}$ be of class $C_{+}^{2}$. For all $0 \leq i \leq n-1$ and any $\alpha \geq 0$,

$$
\begin{aligned}
& \int_{\mathrm{bd} K} \frac{1}{H_{i}^{\alpha}} \mathrm{d} \mathcal{H}^{n-1} \geq \frac{n}{2}\left[\frac{\mathrm{W}_{1}^{\alpha+1}}{\left(\mathrm{~W}_{i+1}+\mathrm{W}_{1} \eta_{1, i, 0}\right)^{\alpha}}+\frac{\mathrm{W}_{1}^{\alpha+1}}{\left(\mathrm{~W}_{i+1}-\mathrm{W}_{1} \eta_{1, i, 0}\right)^{\alpha}}\right] \\
& \int_{\mathrm{bd} K} H_{i}^{\alpha+1} \mathrm{~d} \mathcal{H}^{n-1} \geq \frac{n}{2}\left[\frac{\mathrm{W}_{i+1}^{\alpha+1}}{\left(\mathrm{~W}_{1}+\mathrm{W}_{i+1} \eta_{1, i, i}\right)^{\alpha}}+\frac{\mathrm{W}_{i+1}^{\alpha+1}}{\left(\mathrm{~W}_{1}-\mathrm{W}_{i+1} \eta_{1, i, i}\right)^{\alpha}}\right] .
\end{aligned}
$$

Equality holds in both inequalities if $K=B_{2}^{n}$ (up to dilations).

The lower bounds for $\int_{\mathrm{bd} K} H_{i}^{\alpha+1} \mathrm{~d} \mathcal{H}^{n-1}$ given by (3.9) and (3.12) cannot be compared; indeed, depending on the set, each one of them may be tighter (the same occurs in the case of $\left.\int_{\mathrm{bd} K} 1 / H_{i}^{\alpha} \mathrm{d} \mathcal{H}^{n-1}\right)$. For instance, it can be checked that if $K$ is a convex body such that

$$
\begin{aligned}
\min _{x \in K} \max & \left\{\overline{\mathrm{R}}(K-x)-\frac{\mathrm{W}_{0}}{\mathrm{~W}_{1}}, \frac{\mathrm{W}_{0}}{\mathrm{~W}_{1}}-\overline{\mathrm{r}}(K-x)\right\} \\
& \geq \min _{x \in K} \max \left\{\overline{\mathrm{R}}(K-x)-\frac{\mathrm{W}_{i}}{\mathrm{~W}_{i+1}}, \frac{\mathrm{W}_{i}}{\mathrm{~W}_{i+1}}-\overline{\mathrm{r}}(K-x)\right\},
\end{aligned}
$$

then (3.12) provides a better bound. However, weaker bounds, which are obtained from Theorems 3.3 and 3.4, can be compared. We see it in the following, proving Theorem 1.1.

Proof of Theorem 1.1. First we observe that the fact that the functions $x^{\alpha+1}$ and $1 / x^{\alpha}$ are convex, allows $\eta_{1, i, 0}$ and $\eta_{1, i, i}$ to be replaced by smaller numbers in Theorems 3.3 and 3.4: by (3.4) and (3.5) we immediately get

$$
\begin{aligned}
& \eta_{1, i, 0} \geq \frac{\mathrm{W}_{i} \mathrm{~W}_{1}-\mathrm{W}_{0} \mathrm{~W}_{i+1}}{\mathrm{~W}_{1}^{2} \min _{x \in K}\{\overline{\mathrm{R}}(K-x)-\overline{\mathrm{r}}(K-x)\}}=\frac{\xi_{i}}{n \mathrm{~W}_{1}^{2}} \geq 0 \quad \text { and } \\
& \eta_{1, i, i} \geq \frac{\mathrm{W}_{i} \mathrm{~W}_{1}-\mathrm{W}_{0} \mathrm{~W}_{i+1}}{\mathrm{~W}_{i+1}^{2} \min _{x \in K}\{\overline{\mathrm{R}}(K-x)-\overline{\mathrm{r}}(K-x)\}}=\frac{\xi_{i}}{n \mathrm{~W}_{i+1}^{2}} \geq 0
\end{aligned}
$$


(see the definition of $\xi_{i}$ in Theorem 1.1), and thus (3.12) and (3.9) yield

$$
\begin{gathered}
\int_{\mathrm{bd} K} H_{i}^{\alpha+1} \mathrm{~d} \mathcal{H}^{n-1} \geq \frac{n}{2}\left[\frac{\mathrm{W}_{i+1}^{\alpha+1}}{\left(\mathrm{~W}_{1}+\frac{\xi_{i}}{n \mathrm{~W}_{i+1}}\right)^{\alpha}}+\frac{\mathrm{W}_{i+1}^{\alpha+1}}{\left(\mathrm{~W}_{1}-\frac{\xi_{i}}{n \mathrm{~W}_{i+1}}\right)^{\alpha}}\right], \\
\int_{\mathrm{bd} K} H_{i}^{\alpha+1} \mathrm{~d} \mathcal{H}^{n-1} \geq \frac{n}{2}\left[\frac{\left(\mathrm{W}_{i+1}+\frac{\xi_{i}}{n \mathrm{~W}_{1}}\right)^{\alpha+1}}{\mathrm{~W}_{1}^{\alpha}}+\frac{\left(\mathrm{W}_{i+1}-\frac{\xi_{i}}{n \mathrm{~W}_{1}}\right)^{\alpha+1}}{\mathrm{~W}_{1}^{\alpha}}\right],
\end{gathered}
$$

respectively. But it can be checked that the lower bound in (3.13) is greater than the one in (3.14). Indeed, easy computations allow to see that this fact is equivalent to the inequality

$$
\frac{1}{(1+x)^{\alpha}}+\frac{1}{(1-x)^{\alpha}} \geq(1+x)^{\alpha+1}+(1-x)^{\alpha+1}
$$

for any $\alpha \geq 0$, where

$$
x=\frac{\xi_{i}}{n \mathrm{~W}_{1} \mathrm{~W}_{i+1}}=\frac{\mathrm{W}_{i} \mathrm{~W}_{1}-\mathrm{W}_{0} \mathrm{~W}_{i+1}}{\mathrm{~W}_{1} \mathrm{~W}_{i+1}\left(\overline{\mathrm{R}}\left(K-c_{K}\right)-\overline{\mathrm{r}}\left(K-c_{K}\right)\right)} .
$$

Using again (3.4) and (3.5), we get that

$$
0 \leq \mathrm{W}_{i} \mathrm{~W}_{1}-\mathrm{W}_{0} \mathrm{~W}_{i+1} \leq \mathrm{W}_{1} \mathrm{~W}_{i+1}\left(\overline{\mathrm{R}}\left(K-c_{K}\right)-\overline{\mathrm{r}}\left(K-c_{K}\right)\right),
$$

and therefore, $0 \leq x \leq 1$. Thus, (3.15) is equivalent to see that

$$
f(x)=\frac{\frac{1}{(1+x)^{\alpha}}+\frac{1}{(1-x)^{\alpha}}}{(1+x)^{\alpha+1}+(1-x)^{\alpha+1}} \geq 1
$$

for all $x \in[0,1]$ and any $\alpha \geq 0$. A direct computation shows that the numerator in the first derivative

$$
f^{\prime}(x)=\frac{(1-x)^{2 \alpha+1}[(2 \alpha+1) x+1]-(1+x)^{2 \alpha+1}[1-(2 \alpha+1) x]}{(1-x)^{\alpha+1}(1+x)^{\alpha+1}\left[(1-x)^{\alpha+1}+(1+x)^{\alpha+1}\right]^{2}}
$$

is an increasing function in $x \in[0,1]$, and so non-negative, which ensures that $f(x)$ itself is also increasing and hence $f(x) \geq f(0)=1$, as required. Analogous computations show the lower bound for $\int_{\mathrm{bd} K} 1 / H_{i}^{\alpha} \mathrm{d} \mathcal{H}^{n-1}$.

Remark 3.5. We notice that in the case $i=1$ (mean curvature), the estimates for $\int_{\mathrm{bd} K} H^{n-1} \mathrm{~d} \mathcal{H}^{n-1}$ and $\int_{\mathrm{bd} K} 1 / H \mathrm{~d} \mathcal{H}^{n-1}$ which are obtained from Theorem 1.1 when, respectively, $\alpha=n-2$ and $\alpha=1$ (see Corollary 1.2), improve the known ones for convex hypersurfaces given by (1.1) and (1.2). Indeed, on the one hand,

$$
\frac{1}{n} \frac{|\mathrm{bd} K|^{2} \mathrm{~W}_{2}}{\mathrm{~W}_{2}^{2}-\frac{\xi_{1}^{2}}{|\mathrm{bd} K|^{2}}} \geq \frac{1}{n} \frac{|\mathrm{bd} K|^{2} \mathrm{~W}_{2}}{\mathrm{~W}_{2}^{2}}=n \frac{\mathrm{W}_{1}^{2}}{\mathrm{~W}_{2}} \geq n|K|
$$


because of the Aleksandrov-Fenchel inequality (3.5) for $j=k=1$. On the other hand, since the function $1 / x^{n-2}$ is convex, then

$$
\begin{aligned}
\frac{n^{n-1}}{2}\left[\frac{\mathrm{W}_{2}^{n-1}}{\left(|\mathrm{bd} K|+\frac{\xi_{1}}{\mathrm{~W}_{2}}\right)^{n-2}}+\frac{\mathrm{W}_{2}^{n-1}}{\left(|\mathrm{bd} K|-\frac{\xi_{1}}{\mathrm{~W}_{2}}\right)^{n-2}}\right] & \geq \frac{n^{n-1}}{2} \frac{2 \mathrm{~W}_{2}^{n-1}}{|\mathrm{bd} K|^{n-2}} \\
& =n \frac{\mathrm{W}_{2}^{n-1}}{\mathrm{~W}_{1}^{n-2}} \geq n\left|B_{2}^{n}\right|,
\end{aligned}
$$

where the last inequality follows from the known relations $\mathrm{W}_{j}^{k-i} \geq \mathrm{W}_{i}^{k-j} \mathrm{~W}_{k}^{j-i}$, $0 \leq i<j<k \leq n$, which are also consequences of the Aleksandrov-Fenchel inequality (see e.g. (7.66) in [17]). Similar considerations can be made regarding Theorems 3.3 and 3.4 .

It may also have interest to obtain an estimate for the entropy of the $i$-th mean curvatures. We do it in the following result.

Corollary 3.6. Let $K \in \mathcal{K}_{0}^{n}$ be of class $C_{+}^{2}$. For all $0 \leq i \leq n-1$,

$$
\int_{\mathrm{bd} K} H_{i} \log H_{i} \mathrm{~d} \mathcal{H}^{n-1} \geq-\frac{n}{2} \mathrm{~W}_{i+1} \log \left(\frac{\mathrm{W}_{1}^{2}}{\mathrm{~W}_{i+1}^{2}}-\eta_{1, i, i}^{2}\right) .
$$

Equality holds if $K=B_{2}^{n}$ (up to dilations).

Proof. It is a direct consequence of Theorem 3.2, just taking the convex function $F(x)=\log (1 / x)$ in inequality $(3.8)$.

\section{Radii of curvature of convex bodies}

If $K \in \mathcal{K}_{0}^{n}$ is of class $C_{+}^{2}$, we can consider the $n-1$ principal radii of curvature $r_{1}, \ldots, r_{n-1}$ of $K$ at $u \in \mathbb{S}^{n-1}$, i.e., the eigenvalues of the reverse Weingarten map (see e.g. p. 116 in [17] for a detailed explanation). Then, for $i=1, \ldots, n-1$, we denote by

$$
\varrho_{i}=\mathrm{s}_{i}\left(r_{1}, \ldots, r_{n-1}\right)
$$

the $i$-th normalized elementary symmetric function of the principal radii of curvature, with $\varrho_{0}=1$, and we observe that, properly ordering the indices,

$$
r_{i}(u)=\frac{1}{k_{i}\left(x_{K}(u)\right)}
$$

$i=1, \ldots, n-1$, where $x_{K}(u) \in$ bd $K$ is the unique point of the boundary at which $u$ is the outer normal vector. Moreover, for all $u \in \mathbb{S}^{n-1}, x \in \operatorname{bd} K$,

$$
\varrho_{i}(u)=\frac{H_{n-i-1}}{H_{n-1}}\left(x_{K}(u)\right) \quad \text { and } \quad H_{i}(x)=\frac{\varrho_{n-i-1}}{\varrho_{n-1}}\left(\nu_{K}(x)\right),
$$


and then (see e.g. pp. 296-297 in [17])

$$
\mathrm{W}_{i}=\frac{1}{n} \int_{\mathbb{S}^{n-1}} \varrho_{n-i} \mathrm{~d} \mathcal{H}^{n-1}=\frac{1}{n} \int_{\mathbb{S}^{n-1}} h_{K} \varrho_{n-i-1} \mathrm{~d} \mathcal{H}^{n-1},
$$

$i=0, \ldots, n-1$; the right-hand side identities in (3.6) and (4.1) are usually known in the literature as Minkowski's integral formulae.

An analogous result to Theorem 3.2, now replacing the $i$-th mean curvatures by $\varrho_{i}$, can be obtained. For the sake of brevity we write $\bar{\eta}_{j, k, l}=\eta_{n-j, n-k, n-l}$ (see $(3.3)$ ).

Theorem 4.1. Let $K \in \mathcal{K}_{0}^{n}$ be of class $C_{+}^{2}$. For any convex function $F: I \longrightarrow \mathbb{R}$, $I \subseteq \mathbb{R}$ where all the quantities are defined, and all $0 \leq i \leq n-1$,

$$
\begin{gathered}
\int_{\mathbb{S}^{n-1}}\left(F \circ \varrho_{i}\right) \mathrm{d} \mathcal{H}^{n-1} \geq n\left|B_{2}^{n}\right| \frac{F\left(\frac{\mathrm{W}_{n-i}}{\left|B_{2}^{n}\right|}+\bar{\eta}_{i, 1,1}\right)+F\left(\frac{\mathrm{W}_{n-i}}{\left|B_{2}^{n}\right|}-\bar{\eta}_{i, 1,1}\right)}{2}, \\
\int_{\mathbb{S}^{n-1}}\left(F \circ \frac{1}{\varrho_{i}}\right) \varrho_{i} \mathrm{~d} \mathcal{H}^{n-1} \geq n \mathrm{~W}_{n-i} \frac{F\left(\frac{\left|B_{2}^{n}\right|}{\mathrm{W}_{n-i}}+\bar{\eta}_{i, 1, i+1}\right)+F\left(\frac{\left|B_{2}^{n}\right|}{\mathrm{W}_{n-i}}-\bar{\eta}_{i, 1, i+1}\right)}{2} .
\end{gathered}
$$

Equality holds in both inequalities if $K=B_{2}^{n}$ (up to dilations).

Proof. The first inequality is obtained applying Proposition 1.4 to the probability space $\left(\mathbb{S}^{n-1}, \mathcal{H}^{n-1} /\left(n\left|B_{2}^{n}\right|\right)\right)$ and the functions $\rho=\varrho_{i}$ and $h=h_{K}$. Then, using (4.1), we get $\mathbb{E} \rho=\mathrm{W}_{n-i} /\left|B_{2}^{n}\right|, \mathbb{E} h=\mathrm{W}_{n-1} /\left|B_{2}^{n}\right|$,

$$
\operatorname{Cov}(\rho, h)=\frac{\mathrm{W}_{n-i-1}\left|B_{2}^{n}\right|-\mathrm{W}_{n-i} \mathrm{~W}_{n-1}}{\left|B_{2}^{n}\right|^{2}}
$$

and

$$
\begin{aligned}
\|h-\mathbb{E} h\|_{\infty} & =\sup \left\{\left|h_{K}(u)-\frac{\mathrm{W}_{n-1}}{\left|B_{2}^{n}\right|}\right|: u \in \mathbb{S}^{n-1}\right\} \\
& =\max \left\{\overline{\mathrm{R}}-\frac{\mathrm{W}_{n-1}}{\left|B_{2}^{n}\right|}, \frac{\mathrm{W}_{n-1}}{\left|B_{2}^{n}\right|}-\overline{\mathrm{r}}\right\} .
\end{aligned}
$$

Altogether, and taking into account that all bounds obtained for all possible translations of $K$ are valid, shows the first inequality.

Second inequality is obtained analogously, but now as a consequence of Proposition 2.2 for $\rho=1 / \varrho_{i}, h=h_{K}$ and $g=\varrho_{i} /\left(n \mathrm{~W}_{n-i}\right)$; we notice that, by (4.1), $\int_{\mathbb{S}^{n-1}} g \mathrm{~d} \mathcal{H}^{n-1}=1$. The equality case is trivial.

At this point we would like to mention that analogous results to Theorem 3.3, Theorem 3.4 and Corollary 3.6, for $\varrho_{i}$, can be also proved replacing the convex function $F$ in Theorem 4.1 by the suitable ones. And again, it turns out that it will be not possible to compare the two lower bounds that can be obtained for $\int_{\mathbb{S}^{n-1}} \varrho_{i}^{\alpha+1} \mathrm{~d} \mathcal{H}^{n-1}$, because, depending on the set, each of them might be tighter 
(analogously for $\int_{\mathbb{S}^{n-1}} 1 / \varrho_{i}^{\alpha} \mathrm{d} \mathcal{H}^{n-1}$ ). But as we did in the case of the $H_{i}$ 's, the numbers $\bar{\eta}_{i, 1,1}$ and $\bar{\eta}_{i, 1, i+1}$ may be replaced by smaller numbers in the corresponding results, and thus, comparable weaker bounds can be obtained. We do not repeat all these arguments/results for $\varrho_{i}$ since they are totally analogous to the ones for the $H_{i}$ 's, in order not to enlarge unnecessarily the paper. We just would like to show, as an example, the bound obtained for $\int_{\mathbb{S}^{n-1}} \varrho_{i}^{2} \mathrm{~d} \mathcal{H}^{n-1}$ taking $F(x)=1 / x$ in the second inequality of Theorem 4.1 , as well as the corresponding weaker bound.

Corollary 4.2. Let $K \in \mathcal{K}_{0}^{n}$ be of class $C_{+}^{2}$. For all $0 \leq i \leq n-1$,

$$
\int_{\mathbb{S}^{n-1}} \varrho_{i}^{2} \mathrm{~d} \mathcal{H}^{n-1} \geq \frac{n\left|B_{2}^{n}\right| \mathrm{W}_{n-i}^{2}}{\left|B_{2}^{n}\right|^{2}-\mathrm{W}_{n-i}^{2} \bar{\eta}_{i, 1, i+1}^{2}} \geq \frac{n\left|B_{2}^{n}\right| \mathrm{W}_{n-i}^{2}}{\left|B_{2}^{n}\right|^{2}-\frac{\left(\mathrm{W}_{n-1} \mathrm{~W}_{n-i}-\left|B_{2}^{n}\right| \mathrm{W}_{n-i-1}\right)^{2}}{\mathrm{~W}_{n-i}^{2}\left(\overline{\mathrm{R}}\left(K-c_{K}\right)-\overline{\mathrm{r}}\left(K-c_{K}\right)\right)^{2}}} .
$$

Equality holds, in the first inequality, for $K=B_{2}^{n}$ (up to dilations).

If we intend to provide a lower bound for the integral of $F \circ \varrho_{i}$ in terms of the quermaßintegrals only, an additional hypothesis has to be imposed. The following result is a direct consequence of Theorem 4.1.

Corollary 4.3. Let $K \in \mathcal{K}_{0}^{n}$ be of class $C_{+}^{2}$ and $0 \leq i \leq n-1$ be such that

$$
\max \left\{\overline{\mathrm{R}}-\frac{\mathrm{W}_{n-1}}{\left|B_{2}^{n}\right|}, \frac{\mathrm{W}_{n-1}}{\left|B_{2}^{n}\right|}-\overline{\mathrm{r}}\right\} \leq\left(\frac{\mathrm{W}_{n-1} \mathrm{~W}_{n-i}}{\left|B_{2}^{n}\right|^{2}}-\frac{\mathrm{W}_{n-i-1}}{\left|B_{2}^{n}\right|}\right)^{1 /(i+1)},
$$

and let

$$
\eta=\frac{\mathrm{W}_{n-1} \mathrm{~W}_{n-i}}{\left|B_{2}^{n}\right|^{2}}-\frac{\mathrm{W}_{n-i-1}}{\left|B_{2}^{n}\right|}
$$

Then, for any convex function $F: I \longrightarrow \mathbb{R}, I \subseteq \mathbb{R}$ where all the quantities are defined, we have

$$
\int_{\mathbb{S}^{n-1}}\left(F \circ \varrho_{i}\right) \mathrm{d} \mathcal{H}^{n-1} \geq n\left|B_{2}^{n}\right| \frac{F\left(\frac{\mathrm{W}_{n-i}}{\left|B_{2}^{n}\right|}+\eta^{i /(i+1)}\right)+F\left(\frac{\mathrm{W}_{n-i}}{\left|B_{2}^{n}\right|}-\eta^{i /(i+1)}\right)}{2}
$$

Equality holds for $K=B_{2}^{n}$ (up to dilations).

We observe that the power $1 /(i+1)$ in $(4.2)$ is needed in order to keep the homogeneity in both sides of the inequality, because the $(n-i)$-th quermaßintegral is an homogeneous functional of degree $i$ (see e.g. Theorem 6.13 in [9]).

Remark 4.4. If $K \in \mathcal{K}_{0}^{2}$ is a planar centered convex body of class $C_{+}^{2}$, then assumption (4.2) always holds for $i=1$, as a consequence of the well-known Bonnesen inequality (see e.g. p. 388 in [17]): indeed, (4.2) translates into

$$
\mathrm{p}^{2}-4 \pi \mathrm{A} \geq 4 \pi^{2} \max \left\{\mathrm{R}-\frac{\mathrm{p}}{2 \pi}, \frac{\mathrm{p}}{2 \pi}-\mathrm{r}\right\}^{2}
$$

when $n=2$ and $i=1$, which, depending on the value of the maximum, can be rewritten as either $\mathrm{A}-\mathrm{pR}+\pi \mathrm{R}^{2} \leq 0$ or $\mathrm{A}-\mathrm{pr}+\pi \mathrm{r}^{2} \leq 0$; both inequalities (known as Bonnesen's inequalities) always hold. 
So, it is a natural question to ask wether this condition is also verified in higher dimensions, at least for the class of $C_{+}^{2}$ centered or 0-symmetric convex bodies. Next we answer this question in the negative, and thus, assumption (4.2) cannot be removed. To this end, we need an additional definition. A cap-body (of the ball) is the convex hull of $B_{2}^{n}$ and countably many points such that the line segment joining any pair of those points intersects $B_{2}^{n}$. Cap-bodies are particular cases of the more general defined $p$-tangential bodies for which we refer to Section 2.2 in [17]. It is well known (see [6], Theorem 7.6.17 in [17]) that if $K \in \mathcal{K}_{0}^{n}$ with $B_{2}^{n} \subset K$, then $K$ is a cap-body of $B_{2}^{n}$ if and only if $|K|=\mathrm{W}_{0}=\mathrm{W}_{1}=\cdots=\mathrm{W}_{n-1}$; in particular, $\mathrm{r}=1$.

Proposition 4.5. There exist 0 -symmetric convex bodies $K \in \mathcal{K}_{0}^{n}, n \geq 3$, of class $C_{+}^{2}$, such that, for all $0 \leq i \leq n-1$,

$$
\max \left\{\mathrm{R}-\frac{\mathrm{W}_{n-1}}{\left|B_{2}^{n}\right|}, \frac{\mathrm{W}_{n-1}}{\left|B_{2}^{n}\right|}-\mathrm{r}\right\}>\left(\frac{\mathrm{W}_{n-1} \mathrm{~W}_{n-i}}{\left|B_{2}^{n}\right|^{2}}-\frac{\mathrm{W}_{n-i-1}}{\left|B_{2}^{n}\right|}\right)^{1 /(i+1)} .
$$

Proof. If $i=0$ then any convex body different from a ball verifies the required condition, and so we assume throughout the proof that $1 \leq i \leq n-1$.

Let $K=\operatorname{conv}\left\{B_{2}^{n}, R \mathrm{e}_{1},-R \mathrm{e}_{1}\right\}, R>1$, be a 0-symmetric cap-body, for which $|K|=\mathrm{W}_{0}=\cdots=\mathrm{W}_{n-1}, \mathrm{R}=R$ and $\mathrm{r}=1$. We want to prove that there exists $R>1$ such that, for all $1 \leq i \leq n-1$,

$$
\max \left\{R-\frac{|K|}{\left|B_{2}^{n}\right|}, \frac{|K|}{\left|B_{2}^{n}\right|}-1\right\}>\left(\frac{|K|^{2}}{\left|B_{2}^{n}\right|^{2}}-\frac{|K|}{\left|B_{2}^{n}\right|}\right)^{1 /(i+1)} .
$$

To this end, we first observe that the condition

$$
\frac{|K|}{\left|B_{2}^{n}\right|}-1>\left(\frac{|K|^{2}}{\left|B_{2}^{n}\right|^{2}}-\frac{|K|}{\left|B_{2}^{n}\right|}\right)^{1 /(i+1)}
$$

is equivalent to $\left(|K| /\left|B_{2}^{n}\right|-1\right)^{i}>|K| /\left|B_{2}^{n}\right|$, and hence, if $i \geq 2$, then for $|K|$ large enough (indeed it suffices that $|K| /\left|B_{2}^{n}\right| \geq 3$ ), i.e., for $R$ large enough, condition (4.4) holds.

So we assume $i=1$, and we have to show that there exists $R>1$ such that $R-|K| /\left|B_{2}^{n}\right|>\left(|K|^{2} /\left|B_{2}^{n}\right|^{2}-|K| /\left|B_{2}^{n}\right|\right)^{1 / 2}$, or equivalently, such that

$$
|K|<\left|B_{2}^{n}\right| \frac{R^{2}}{2 R-1} .
$$

It can be seen (see e.g. [10]) that the volume of such a cap-body is given by

$$
|K|=2\left|B_{2}^{n-1}\right|\left(\frac{\left(R^{2}-1\right)^{(n+1) / 2}}{n R^{n}}+\int_{0}^{1 / R}\left(1-x^{2}\right)^{(n-1) / 2} \mathrm{~d} x\right),
$$

and hence we have to show that there exists $R>1$ such that

$$
\frac{2 R-1}{R^{2}}\left(\frac{\left(R^{2}-1\right)^{(n+1) / 2}}{n R^{n}}+\int_{0}^{1 / R}\left(1-x^{2}\right)^{(n-1) / 2} \mathrm{~d} x\right)<\frac{\left|B_{2}^{n}\right|}{2\left|B_{2}^{n-1}\right|}
$$


for all $n \geq 3$. If $n=3$ the above condition reduces to $R^{2}+1>2 R$, which always holds. Moreover, $\int_{0}^{1 / R}\left(1-x^{2}\right)^{(n-1) / 2} \mathrm{~d} x$ is decreasing in $R$, and hence

$$
\int_{0}^{1 / R}\left(1-x^{2}\right)^{(n-1) / 2} \mathrm{~d} x<\int_{0}^{1}\left(1-x^{2}\right)^{(n-1) / 2} \mathrm{~d} x=\frac{\left|B_{2}^{n}\right|}{2\left|B_{2}^{n-1}\right|}
$$

(see (3.2)). Therefore, it suffices to see that

$$
\frac{2 R-1}{R^{2}}\left(\frac{\left(R^{2}-1\right)^{(n+1) / 2}}{n R^{n}}+\frac{\left|B_{2}^{n}\right|}{2\left|B_{2}^{n-1}\right|}\right)<\frac{\left|B_{2}^{n}\right|}{2\left|B_{2}^{n-1}\right|}
$$

for some $R>1$, or equivalently,

$$
\frac{(2 R-1)(R+1)^{(n+1) / 2}(R-1)^{(n-3) / 2}}{R^{n}}<\frac{n}{2} \frac{\left|B_{2}^{n}\right|}{\left|B_{2}^{n-1}\right|},
$$

which is trivially true, for all $n \geq 4$, if $R>1$ is sufficiently close to 1 . It proves (4.5) and hence (4.4).

Thus we have shown that there exists a 0 -symmetric cap-body $K \in \mathcal{K}_{0}^{n}$ satisfying inequality (4.3). Since every convex body can be approximated by convex bodies of class $C_{+}^{2}$ (with respect to the Hausdorff metric, see Section 3.4 in [17]), we can find, on the one hand, a sequence $\left(K_{j}\right)_{j \in \mathbb{N}} \subset \mathcal{K}_{0}^{n}$ of $C_{+}^{2}$ sets with $\lim _{j \rightarrow \infty} K_{j}=K$. Moreover, the sequence $\left(K_{j}^{c}=\left(K_{j}-K_{j}\right) / 2\right)_{j \in \mathbb{N}}$, which is also formed by $C_{+}^{2}$ convex bodies, satisfies that

$$
\lim _{j \rightarrow \infty} \frac{1}{2}\left(K_{j}-K_{j}\right)=\frac{1}{2}(K-K)=K,
$$

because $K$ is 0 -symmetric. Hence, we have a sequence $\left(K_{j}^{c}\right)_{j \in \mathbb{N}}$ of 0 -symmetric convex bodies of class $C_{+}^{2}$ with limit $K$. On the other hand, since the quermaßintegrals, circumradius and inradius are continuous functionals (with respect to the Hausdorff metric, see e.g. Theorem 6.13 in [9]), then we can conclude that there exists a 0 -symmetric convex body $K_{j}^{c} \in \mathcal{K}_{0}^{n}$ of class $C_{+}^{2}$, for $j$ large enough, satisfying (4.3) for all $0 \leq i \leq n-1$.

\section{Radii of curvature and roots of Steiner polynomials}

On the one hand, in [11], the roots of the Steiner polynomial of a convex body $K$ (cf. (3.1)), considered as a formal polynomial in a complex variable $z \in \mathbb{C}$, are related to the maximum and minimum value of its principal radii of curvature. On the other hand, in [8], lower estimates for the integrals of convex functions of the curvature of a smooth bounded planar convex curve are shown in terms of the roots of the Steiner polynomial of the region enclosed by the curve.

Following this idea, in this section we are going to relate the integral of a transformation of the $i$-th normalized elementary symmetric function of the principal radii of curvature of a $C_{+}^{2}$ convex body $K$, to the roots of its the Steiner polynomial. 
To this end, for $K \in \mathcal{K}_{0}^{n}$, we denote by $f_{K}(z)=\sum_{i=0}^{n}\left(\begin{array}{c}n \\ i\end{array}\right) \mathrm{W}_{i} z^{i}$ the Steiner polynomial of $K$. Moreover, let $\gamma_{1}, \ldots, \gamma_{n} \in \mathbb{C}$ be the roots of $f_{K}(z)$ and, for the sake of brevity, we write $\mathrm{s}_{i}(\gamma)=\mathrm{s}_{i}\left(\gamma_{1}, \ldots, \gamma_{n}\right)$.

Proposition 5.1. Let $K \in \mathcal{K}_{0}^{n}$ be of class $C_{+}^{2}$, with $c_{K}=0$ and $K \neq B_{2}^{n}(r)$ for any $r>0$. For any convex function $F: I \longrightarrow \mathbb{R}, I \subseteq \mathbb{R}$ where all the quantities are defined, and all $0 \leq i \leq n-1$,

$$
\begin{aligned}
& \int_{\mathbb{S}^{n-1}}\left(F \circ \varrho_{i}\right) \mathrm{d} \mathcal{H}^{n-1} \geq \frac{n\left|B_{2}^{n}\right|}{2}[ F\left(\left|\mathrm{~s}_{i}(\gamma)\right|+\frac{\left|\mathrm{s}_{i}(\gamma) \mathrm{s}_{1}(\gamma)\right|-\left|\mathrm{s}_{i+1}(\gamma)\right|}{\overline{\mathrm{R}}-\overline{\mathrm{r}}}\right) \\
&+F\left(\left|\mathrm{~s}_{i}(\gamma)\right|-\frac{\left|\mathrm{s}_{i}(\gamma) \mathrm{s}_{1}(\gamma)\right|-\left|\mathrm{s}_{i+1}(\gamma)\right|}{\overline{\mathrm{R}}-\overline{\mathrm{r}})] .}\right.
\end{aligned}
$$

Proof. From $f_{K}(z)=\left|B_{2}^{n}\right| \prod_{i=1}^{n}\left(z-\gamma_{i}\right)$ we get

$$
\left(\begin{array}{c}
n \\
i
\end{array}\right) \mathrm{W}_{n-i}=(-1)^{i}\left|B_{2}^{n}\right| \sigma_{i}\left(\gamma_{1}, \ldots, \gamma_{n}\right), \quad \text { i.e., } \quad \frac{\mathrm{W}_{n-i}}{\left|B_{2}^{n}\right|}=\left|\mathrm{s}_{i}(\gamma)\right| .
$$

Then, using Theorem 4.1, together with (3.4), we get the result.

Remark 5.2. When $i=n-1$, the argument of the function $F$ in the above expression can be expressed in terms of the real parts of the roots and their inverses: denoting by $\operatorname{Re} z$ the real part of a complex number $z \in \mathbb{C}$, we get

$$
\begin{aligned}
\left|\mathrm{s}_{n-1}(\gamma)\right| & +\frac{\left|\mathrm{s}_{n-1}(\gamma) \mathrm{s}_{1}(\gamma)\right|-\left|\mathrm{s}_{n}(\gamma)\right|}{\overline{\mathrm{R}}-\overline{\mathrm{r}}} \\
& =\left|\mathrm{s}_{n}(\gamma)\right|\left[\left|\frac{\mathrm{s}_{n-1}(\gamma)}{\mathrm{s}_{n}(\gamma)}\right|+\frac{\left|\frac{\mathrm{s}_{n-1}(\gamma)}{\mathrm{s}_{n}(\gamma)} \mathrm{s}_{1}(\gamma)\right|-1}{\overline{\mathrm{R}}-\overline{\mathrm{r}}}\right] \\
& =\left|\mathrm{s}_{n}(\gamma)\right|\left[\left|\mathrm{s}_{1}\left(\frac{1}{\gamma}\right)\right|+\frac{\left|\mathrm{s}_{1}\left(\frac{1}{\gamma}\right) \mathrm{s}_{1}(\gamma)\right|-1}{\overline{\mathrm{R}}-\overline{\mathrm{r}}}\right] \\
& =\left|\gamma_{1} \cdots \gamma_{n}\right|\left[\left|\sum_{i=1}^{n} \operatorname{Re} \frac{1}{\gamma_{i}}\right|+\frac{\left|\sum_{i=1}^{n} \operatorname{Re} \frac{1}{\gamma_{i}}\right|\left|\sum_{i=1}^{n} \operatorname{Re} \gamma_{i}\right|-1}{\overline{\mathrm{R}}-\overline{\mathrm{r}}}\right] .
\end{aligned}
$$

For particular families of sets, the bounds provided in Sections 3 and 4 can be expressed in a more convenient way. We show here a couple of examples. Similar results can be obtained for all $H_{i}, i=2, \ldots, n-1$.

Proposition 5.3. Let $K \in \mathcal{K}_{0}^{n}$ be of class $C_{+}^{2}$ such that $|K|=\mathrm{W}_{1}=\mathrm{W}_{2}$. Then, for any $\alpha \geq 0$,

$$
\int_{\mathrm{bd} K} H^{\alpha+1} \mathrm{~d} \mathcal{H}^{n-1} \geq n|K| \quad \text { and } \quad \int_{\mathrm{bd} K} \frac{1}{H^{\alpha}} \mathrm{d} \mathcal{H}^{n-1} \geq n|K| .
$$

Equality holds in both inequalities if $K=B_{2}^{n}$ (up to dilations). 
On a rather different tack, a convex body $K \in \mathcal{K}_{0}^{n}$ is said to have constant width if it has the same width $\omega_{K}(u)=h_{K}(u)+h_{K}(-u)$ in all directions $u \in \mathbb{S}^{n-1}$. Constant width sets have been intensively studied along the last century. In the plane they are well known, whereas the situation becomes much more complicated in dimension $n \geq 3$.

It is well known (see e.g. p. 68 in [3]) that if $K \in \mathcal{K}_{0}^{n}$ has constant width b, then the inball and the circumball of $K$ are concentric and $\mathrm{R}+\mathrm{r}=\mathrm{b}$. Thus, assuming that the origin is the incenter of $K$, we have $\overline{\mathrm{R}}=\mathrm{R}$ and $\overline{\mathrm{r}}=\mathrm{r}$. Moreover,

$$
\mathrm{b}\left(1-\sqrt{\frac{n}{2(n+1)}}\right) \leq \mathrm{r} \leq \mathrm{R} \leq \mathrm{b} \sqrt{\frac{n}{2(n+1)}}
$$

(see e.g. p. 68 and (7.3) in [3]). For a nice and thorough survey on convex bodies of constant width see [3].

Using Theorem 4.1, the following type of results can be obtained for constant width sets. For the sake of brevity we write $c_{n}=\sqrt{2 n /(n+1)}-1$.

Theorem 5.4. Let $K \in \mathcal{K}_{0}^{n}$ be a $C_{+}^{2}$ constant width set of width $\mathrm{b}$. Then, for any convex function $F: I \longrightarrow \mathbb{R}, I \subseteq \mathbb{R}$ where all the quantities are defined, and all $0 \leq i \leq n-1$,

$$
\begin{aligned}
\int_{\mathbb{S}^{n-1}}\left(F \circ \varrho_{i}\right) \mathrm{d} \mathcal{H}^{n-1} \geq \frac{n\left|B_{2}^{n}\right|}{2}[ & F\left(\frac{\mathrm{W}_{n-i}}{\left|B_{2}^{n}\right|}+\frac{\mathrm{bW}_{n-i}-2 \mathrm{~W}_{n-i-1}}{c_{n}\left|B_{2}^{n}\right| \mathrm{b}}\right) \\
& \left.+F\left(\frac{\mathrm{W}_{n-i}}{\left|B_{2}^{n}\right|}-\frac{\mathrm{bW}_{n-i}-2 \mathrm{~W}_{n-i-1}}{c_{n}\left|B_{2}^{n}\right| \mathrm{b}}\right)\right] .
\end{aligned}
$$

Moreover,

$$
\begin{aligned}
& \int_{\mathbb{S}^{n-1}}\left(F \circ \varrho_{i}\right) \mathrm{d} \mathcal{H}^{n-1} \geq \frac{n\left|B_{2}^{n}\right|}{2} {\left[F\left(\frac{c_{n}+1}{c_{n}}\left|\mathrm{~s}_{i}(\gamma)\right|-\frac{2\left|\mathrm{~s}_{i+1}(\gamma)\right|}{c_{n} \mathrm{~b}}\right)\right.} \\
&\left.+F\left(\frac{c_{n}-1}{c_{n}}\left|\mathrm{~s}_{i}(\gamma)\right|+\frac{2\left|\mathrm{~s}_{i+1}(\gamma)\right|}{c_{n} \mathrm{~b}}\right)\right] .
\end{aligned}
$$

Equality holds in both inequalities if $K=B_{2}^{n}$.

Proof. Since $\mathrm{W}_{n-1} /\left|B_{2}^{n}\right|=\mathrm{b} / 2$ and $\mathrm{R}+\mathrm{r}=\mathrm{b}$, using (5.2) we get

$$
\frac{\mathrm{W}_{n-1}}{\left|B_{2}^{n}\right|}-\mathrm{r}=\frac{\mathrm{b}}{2}-\mathrm{r}=\frac{\mathrm{b}}{2}-(\mathrm{b}-\mathrm{R})=\mathrm{R}-\frac{\mathrm{b}}{2} \leq \frac{\mathrm{b}}{2}\left(\sqrt{\frac{2 n}{n+1}}-1\right),
$$

and therefore

$$
\begin{aligned}
\bar{\eta}_{i, 1,1} & =\frac{\mathrm{W}_{n-i} \mathrm{~W}_{n-1}-\left|B_{2}^{n}\right| \mathrm{W}_{n-i-1}}{\left|B_{2}^{n}\right|^{2} \max \left\{\mathrm{R}-\frac{\mathrm{W}_{n-1}}{\left|B_{2}^{n}\right|}, \frac{\mathrm{W}_{n-1}}{\left|B_{2}^{n}\right|}-\mathrm{r}\right\}}=\frac{\mathrm{bW}_{n-i}-2 \mathrm{~W}_{n-i-1}}{2\left|B_{2}^{n}\right|\left(\mathrm{R}-\frac{\mathrm{b}}{2}\right)} \\
& \geq \frac{\mathrm{bW}_{n-i}-2 \mathrm{~W}_{n-i-1}}{c_{n}\left|B_{2}^{n}\right| \mathrm{b}} .
\end{aligned}
$$

Using the above bound, the first inequality is a direct consequence of Theorem 4.1; the second inequality follows from the first one and (5.1). 


\section{Radial function and dual quermaßintegrals}

A non-empty set $S \subset \mathbb{R}^{n}$ is called starshaped (with respect to 0 ) if the line segment $[0, x] \subseteq S$ for all $x \in S$. For a compact starshaped set $K$, the radial function is defined as

$$
\rho_{K}(u)=\max \{\lambda \geq 0: \lambda u \in K\}, \quad u \in \mathbb{R}^{n} \backslash\{0\},
$$

and clearly, $\rho_{K}(u) u \in \operatorname{bd} K$. We will denote by $\mathcal{S}_{0}^{n}$ the family of all compact starshaped sets in $\mathbb{R}^{n}$ having the origin as an interior point.

Dual quermaßintegrals (and dual mixed volumes) were introduced by Lutwak in [13], being the starting point for the development of the nowadays known as dual Brunn-Minkowski theory (see e.g. Section 9.3 in [17]). The dual quermaßintegral of order $i, i=0, \ldots, n$, of $K \in \mathcal{S}_{0}^{n}$ is defined as

$$
\widetilde{\mathrm{W}}_{i}(K)=\frac{1}{n} \int_{\mathbb{S}^{n-1}} \rho_{K}^{n-i} \mathrm{~d} \mathcal{H}^{n-1},
$$

which is monotonous and homogeneous of degree $n-i$ (see e.g. Section A.7 in [7]), although not translation invariant. In particular, the use of spherical coordinates immediately yields $\widetilde{\mathrm{W}}_{0}(K)=|K|$, whereas $\widetilde{\mathrm{W}}_{n}(K)=\left|B_{2}^{n}\right|$ and $2 \widetilde{\mathrm{W}}_{n-1}(K) /\left|B_{2}^{n}\right|$ is the average length of chords of $K$ through the origin. Moreover, if $K \in \mathcal{K}_{0}^{n}$, then $\widetilde{\mathrm{W}}_{i}(K) \leq \mathrm{W}_{i}(K)$ for all $i=0, \ldots, n$ (see [13]).

Let $K \in \mathcal{S}_{0}^{n}$. Again, for the sake of brevity, we will write $\widetilde{\mathrm{W}}_{i}=\widetilde{\mathrm{W}}_{i}(K)$, and for any $0 \leq j, k, l \leq n$ with $j+k \leq n$, let

$$
\begin{aligned}
& \widetilde{\eta}_{j, k, l}=\frac{|K|\left|B_{2}^{n}\right|-\widetilde{\mathrm{W}}_{n-j} \widetilde{\mathrm{W}}_{j}}{\widetilde{\mathrm{W}}_{n-l}^{2} \max \left\{\overline{\mathrm{R}}^{n-j}-\frac{\widetilde{\mathrm{W}}_{k}}{\mathrm{~W}_{n-j+k}}, \frac{\widetilde{\mathrm{W}}_{k}}{\mathrm{~W}_{n-j+k}}-\overline{\mathrm{r}}^{n-j}\right\}} \text { if } K \neq B_{2}^{n}(r), r>0, \\
& \widetilde{\eta}_{j, k, l}=0 \quad \text { if } K=B_{2}^{n}(r) \text { for some } r>0,
\end{aligned}
$$

where $\overline{\mathrm{R}}, \overline{\mathrm{r}}$ are defined analogously to the convex case. From the monotonicity of the dual quermaßintegrals we get $\overline{\mathrm{r}}^{n-j} \widetilde{\mathrm{W}}_{n-j+k} \leq \widetilde{\mathrm{W}}_{k} \leq \overline{\mathrm{R}}^{n-j} \widetilde{\mathrm{W}}_{n-j+k}$ (cf. (3.4)), and so the above maximum is always positive. This, together with the relation $|K|\left|B_{2}^{n}\right| \geq \widetilde{\mathrm{W}}_{n-j} \widetilde{\mathrm{W}}_{j}$ (a consequence of the dual Aleksandrov-Fenchel inequalities, see e.g. Theorem 2 in [13]), ensures that $\widetilde{\eta}_{j, k, l} \geq 0$.

Theorem 6.1. Let $K \in \mathcal{S}_{0}^{n}$. For any convex function $F: I \longrightarrow \mathbb{R}, I \subseteq \mathbb{R}$ where all the quantities are defined, and all $0 \leq i \leq n$,

$$
\begin{gathered}
\int_{\mathbb{S}^{n-1}}\left(F \circ \rho_{K}^{i}\right) \mathrm{d} \mathcal{H}^{n-1} \geq n\left|B_{2}^{n}\right| \frac{F\left(\frac{\widetilde{\mathrm{W}}_{n-i}}{\left|B_{2}^{n}\right|}+\widetilde{\eta}_{i, i, 0}\right)+F\left(\frac{\widetilde{\mathrm{W}}_{n-i}}{\left|B_{2}^{n}\right|}-\widetilde{\eta}_{i, i, 0}\right)}{2}, \\
\int_{\mathbb{S}^{n-1}}\left(F \circ \frac{1}{\rho_{K}^{i}}\right) \rho_{K}^{i} \mathrm{~d} \mathcal{H}^{n-1} \geq n \widetilde{\mathrm{W}}_{n-i} \frac{F\left(\frac{\left|B_{2}^{n}\right|}{\widetilde{\mathrm{W}}_{n-i}}+\widetilde{\eta}_{i, 0, i}\right)+F\left(\frac{\left|B_{2}^{n}\right|}{\widehat{\mathrm{W}}_{n-i}}-\widetilde{\eta}_{i, 0, i}\right)}{2} .
\end{gathered}
$$

Equality holds in both inequalities if $K=B_{2}^{n}$ (up to dilations). 
Proof. In order to prove (6.1) we apply Proposition 1.4 to the probability space $\left(\mathbb{S}^{n-1}, \mathcal{H}^{n-1} /\left(n\left|B_{2}^{n}\right|\right)\right)$ and the functions $\rho=\rho_{K}^{i}$ and $h=\rho_{K}^{n-i}$. Then, by (6.1), we get $\mathbb{E} \rho=\widetilde{\mathrm{W}}_{n-i} /\left|B_{2}^{n}\right|, \mathbb{E} h=\widetilde{\mathrm{W}}_{i} /\left|B_{2}^{n}\right|$ and

$$
\operatorname{Cov}(\rho, h)=\frac{|K|\left|B_{2}^{n}\right|-\widetilde{\mathrm{W}}_{n-i} \widetilde{\mathrm{W}}_{i}}{\left|B_{2}^{n}\right|^{2}} .
$$

Moreover, since $\rho_{K}(u) u \in \operatorname{bd} K$, we have

$$
\begin{aligned}
\|h-\mathbb{E} h\|_{\infty} & =\sup \left\{\left|\rho_{K}(u)^{n-i}-\frac{\widetilde{\mathrm{W}}_{i}}{\left|B_{2}^{n}\right|}\right|: u \in \mathbb{S}^{n-1}\right\} \\
& =\max \left\{\overline{\mathrm{R}}^{n-i}-\frac{\widetilde{\mathrm{W}}_{i}}{\left|B_{2}^{n}\right|}, \frac{\widetilde{\mathrm{W}}_{i}}{\left|B_{2}^{n}\right|}-\overline{\mathrm{r}}^{n-i}\right\} .
\end{aligned}
$$

Altogether shows the first inequality.

Second inequality is obtained analogously, but now as a consequence of Proposition 2.2 for $\rho=1 / \rho_{K}^{i}, h=\rho_{K}^{n-i}$ and $g=\rho_{K}^{i} /\left(n \widetilde{\mathrm{W}}_{n-i}\right)$. Equality case is trivial.

Remark 6.2. We observe that, since (6.1) can be defined for any $i \in \mathbb{R}$, Theorem 6.1 holds true for all $i \in \mathbb{R}$, just properly defining the values $\widetilde{\eta}_{i, j, k}$. Moreover, taking $F(x)=x^{\alpha+1}$ or $F(x)=1 / x^{\alpha}$ for suitable powers $\alpha \geq 0$, and considering (6.1) defined for any $i \in \mathbb{R}$, new inequalities relating the dual quermaßintegrals with the in- and outer radii can be obtained.

Acknowledgements: The authors would like to thank the anonymous referee for the very valuable comments and suggestions.

\section{References}

[1] Andrews, B. AND McCoy, J.: Convex hypersurfaces with pinched principal curvatures and flow of convex hypersurfaces by high powers of curvature. Trans. Amer. Math. Soc. 364 (2012), no. 7, 3427-3447.

[2] BÁrÁny, I.: On the minimal ring containing the boundary of a convex body. Acta Sci. Math. 52 (1988), 93-100.

[3] Chakerian, G. D. and Groemer, H.: Convex bodies of constant width. In Convexity and its applications., 49-96. Birkhuser Verlag, Basel-Boston, Mass., ed. P. M. Gruber and J. M. Wills, 1983.

[4] Chen, B.-Y.: On the total curvature of immersed manifolds. I. An inequality of Fenchel-Borsuk-Willmore. Amer. J. Math. 93 (1971), 148-162.

[5] Chen, B.-Y.: Geometry of submanifolds and its applications. Science University of Tokyo, Tokyo, 1981.

[6] Favard, J.: Sur les corps convexes. J. Math. Pures Appl. 12 (1933), no. 9, 219-282.

[7] Gardner, R. J.: Geometric tomography. Cambridge University Press, Cambridge, Encyclopedia of Mathematics and its Applications 58, 2nd edition, 2006. 
[8] Green, M. And Osher, S.: Steiner polynomials, Wulff flows, and some new isoperimetric inequalities for convex plane curves. Asian J. Math. 3 (1999), no. 3, 659-676.

[9] Gruber, P. M.: Convex and Discrete Geometry. Springer, Berlin Heidelberg, 2007.

[10] Hernández Cifre, M.A., Pastor, J.A., Salinas, G. and Segura Gomis, S.: Complete systems of inequalities for centrally symmetric convex sets in the $n$ dimensional space. Arch. Inequal. Appl. 1 (2003), no. 2, 155-167.

[11] Jetter, M.: Bounds on the roots of the Steiner polynomial. Adv. Geom. 11 (2011), 313-319.

[12] Li, P. And YAU, S. T.: A new conformal invariant and its applications to the Willmore conjecture and the first eigenvalue of compact surfaces. Invent. Math. 69 (1982), no. 2, 269-291.

[13] Lutwak, E.: Dual mixed volumes. Pacific J. Math. 58 (1975), no. 2, 531-538.

[14] Marques, F. C. And Neves, A.: Min-Max Theory and the Willmore conjecture. Ann. Math. 179 (2014), no.2, 683-782.

[15] Ritoré, M. and Sinestrari, C.: Mean curvature flow and isoperimetric inequalities., Birkhäuser Verlag, Basel, Advanced Courses in Mathematics, ed. V. Miquel and J. Porti, CRM Barcelona, 2010.

[16] Ros, A.: Compact hypersurfaces with constant higher order mean curvatures. Rev. Mat. Iberoamericana 3 (1987), no. 3-4, 447-453.

[17] Schneider, R.: Convex bodies: The Brunn-Minkowski theory. Cambridge University Press, Cambridge, 2nd expanded edition, 2014.

[18] Steiner, J.: Über parallele Flächen. Monatsber. Preuss. Akad. Wiss. (1840), 114118, [Ges. Werke, Vol II (Reimer, Berlin, 1882) 245-308].

[19] Webster, R.: Convexity. Oxford University Press, New York, 1994.

[20] Willmore, T. J.: Mean curvature of immersed surfaces. An. Şti. Univ. "All. I. Cuza" Iaşi Secţ. I a Mat. 14 (1968), 99-103.

Received ??

DAvid Alonso-GutiÉrRez: Departamento de Matemáticas, Universidad de Zaragoza, C/ Pedro Cerbuna 12, 50009-Zaragoza, Spain

E-mail: alonsod@unizar.es

María A. Hernández Cifre: Departamento de Matemáticas, Universidad de Murcia, Campus de Espinardo, 30100-Murcia, Spain

E-mail: mhcifre@um.es

Antonio R. Martínez Fernández: Departamento de Matemáticas, Universidad de Murcia, Campus de Espinardo, 30100-Murcia, Spain

E-mail: antonioroberto.martinez@um.es

This work is a result of the activity developed within the framework of the Programme in Support of Excellence Groups of the Región de Murcia, Spain, by Fundación Séneca, Science and Technology Agency of the Región de Murcia.

First author was partially supported by MINECO project MTM2013-42105-P, and BANCAJA project P1-1B2014-35. Second and third authors were partially supported by MINECO/FEDER projects MTM2012-34037 and MTM2015-65430-P, Spain, and Fundación Séneca project reference 19901/GERM/15, Spain. 